Plutonium Solution Analyzer

Donald A. Burns
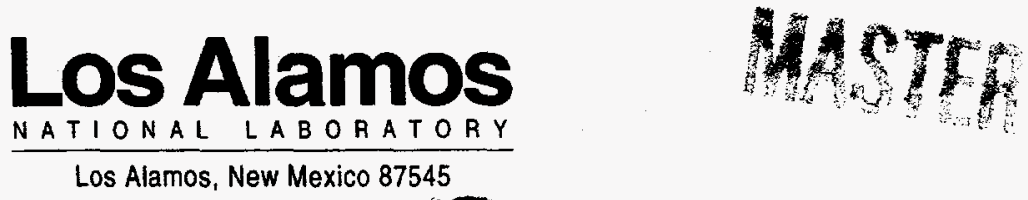


\section{DISCLAIMER}

This report was prepared as an account of work sponsored by an agency of the United States Government. Neither the United States Government nor any agency thereof, nor any of their employees, make any warranty, express or implied, or assumes any legal liability or responsibility for the accuracy, completeness, or usefulness of any information, apparatus, product, or process disclosed, or represents that its use would not infringe privately owned rights. Reference herein to any specific commercial product, process, or service by trade name, trademark, manufacturer, or otherwise does not necessarily constitute or imply its endorsement, recommendation, or favoring by the United States Government or any agency thereof. The views and opinions of authors expressed herein do not necessarily state or reflect those of the United States Government or any agency thereof. 


\section{DISCLAIMER}

Portions of this document may be illegible in electronic image products. Images are produced from the best available original document. 


\title{
PLUTONIUM SOLUTION ANALYZER
}

by

Donald A. Burns

\begin{abstract}
A fully automated analyzer has been developed for plutonium solutions. It was assembled from several commercially available modules, is based upon segmented flow analysis, and exhibits precision about an order of magnitude better than commercial units $(0.5 \%-0.05 \%$ RSD). The system was designed to accept unmeasured, untreated liquid samples in the concentration range 40-240 $\mathrm{g} / \mathrm{L}$ and produce a report with sample identification, sample concentrations, and an abundance of statistics. Optional hydraulics can accommodate samples in the concentration range $0.4-4.0 \mathrm{~g} / \mathrm{L}$. Operating at a typical rate of 30 to 40 samples per hour, it consumes only $0.074 \mathrm{~mL}$ of each sample and standard, and generates waste at the rate of about $1.5 \mathrm{~mL}$ per minute. No radioactive material passes through its multichannel peristaltic pump (which remains outside the glovebox, uncontaminated) but rather is handled by a 6-port, 2-position chromatography-type loop valve. An accompanying computer is programmed in QuickBASIC 4.5 to provide both instrument control and data reduction. The program is truly user-friendly and communication between operator and instrument is via computer screen displays and keyboard. Two important issues which have been addressed are waste minimization and operator safety (the analyzer can run in the absence of an operator, once its autosampler has been loaded).
\end{abstract}

\section{INTRODUCTION}

A high-precision analyzer for plutonium ( $\mathrm{Pu}$ ) solutions has been assembled from several commercially available modules. The analyzer was designed to handle radioactive or hazardous materials inside a glovebox, and samples come in direct contact with a minimum of modules. Samples as small as $0.074 \mathrm{~mL}$ containing $40-240 \mathrm{~g} / \mathrm{L} \mathrm{Pu}$ can be analyzed at the rate of 40 per hour with 
precision in the range $0.5 \%$ to $0.05 \% \mathrm{RSD}$ (relative standard deviation). Waste is generated at the rate of about $1.5 \mathrm{~mL}$ per minute.

\section{CHEMISTRY}

Plutonium in aqueous solutions can exist in several oxidation states (III, IV, and VI are common) ${ }^{1}$ and is usually converted to its lowest or highest state for spectrophotometry. We followed procedures developed by Tober and Russell ${ }^{2}$ in 1959 and by Burney and Tober ${ }^{3}$ in 1965, in which ascorbic acid in $2 \mathrm{~N}$ hydrochloric acid $(\mathrm{HCl})$ reduces it to $\mathrm{Pu}+3$. These procedures in use at Los Alamos National Laboratory were reported in 1984 by Harmon, Hahn, and Marshall. 4 The reaction goes to completion in less than 15 seconds at room temperature. The resulting spectrum has two peaks, along with the valley between them, and both preceding and following baselines, that are used for quantitation. Figure 1 shows a typical tracing with the five important wavelengths $(516,560,586,600$, and $640 \mathrm{~nm})$ identified with letters (A through $E$, respectively). The absorbances at these five wavelengths produce four absorbance differences (B-A, B-C, D-C, and D-E) that are then used for both calibration with standards and quantitation of samples.

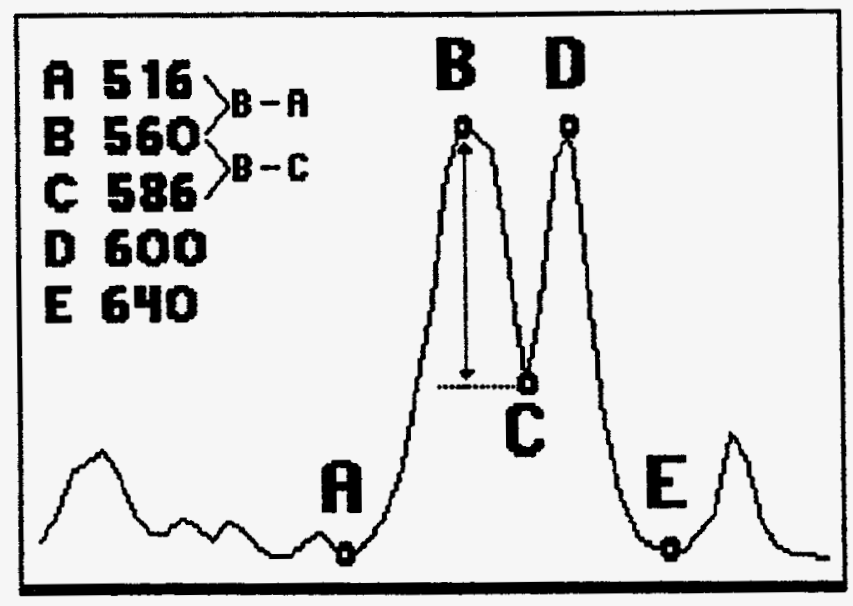

Figure 1. Plutonium spectrum.

This algorithm, long used at Los Alamos National Laboratory, was studied in considerable detail and confirmed in 1988 by Wangen, Phillips, and Walker. ${ }^{5}$

\section{PRINCIPLE OF OPERATION}

The procedures in a chemical analysis may be performed manually or may be automated. Two kinds of automation exist: continuous flow (CF) and batch procedures. Within CF one finds segmented flow analysis (SFA) and flow 
injection analysis (FIA). Technicon Instruments Corporation developed SFA in the early 1950s, and Technicon's AutoAnalyzer was used primarily for clinical chemistry. The technique quickly found its way into the industrial laboratory because of its simplicity and versatility. All of these unit operations can be performed in SFA:

$\begin{array}{lll}\text { aliquoting } & \text { heating } & \text { phase separation } \\ \text { mixing } & \text { cooling } & \text { precipitation } \\ \text { dilution } & \text { filtration } & \text { distillation } \\ \text { reagent addition } & \text { dialysis } & \text { evaporation to } \\ \text { incubation } & \text { solvent extraction } & \text { dryness }\end{array}$

The five in the left-hand column are used in the Pu analyzer described herein. SFA was selected to maximize both analytical rate and precision, and to allow for upgrading to multiple simultaneous analyses (è.g., acidity and additional anions or cations).

In operation, an air-segmented carrier stream is generated with a multichannel peristaltic pump, and pump tubes of various sizes control what is added to the carrier stream, at what rate, and when.

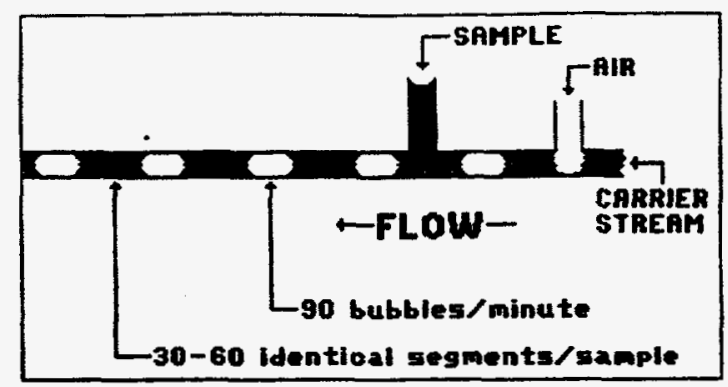

Figure 2. Carrier stream generation

In this system, the carrier stream (see Figure 2) is water (used as a diluent) to which air bubbles are added at the rate of 90 per minute, thereby producing a segmented stream. Ascorbic acid (the reagent) is added continuously, and samples or standards (alternating with a wash solution) are added at defined intervals. All solutions contain a wetting agent (Brij-35, 8 drops per liter).

Helical mixing coils of sufficient length are included to ensure that the reaction goes to completion and that a homogeneous solution is available at the detector. Mixing coil dimensions are such that the individual liquid segments are repeatedly inverted as they worm their way through the helix, and this action promotes rapid and efficient mixing. By the time the reaction mixture reaches the flowcell, color has developed and stabilized. 
The manufacturers of SFA hardware have taken two approaches to flowcell hydraulics. Initially, the streams were debubbled so that no air would ever enter the flowcell and perturb the passage of light through its optical path. To accomplish this, $80 \%$ to $90 \%$ of the liquid is pumped through the flowcell, and the remainder goes to waste along with the segmenting air. More recently, the bubble is permitted to pass through the flowcell (hereinafter referred to as bubble through the flowcell) Appropriate software performs "electronic debubbling," wherein the interruption of the light beam caused by the bubbles is ignored and absorbance data are obtained only when the light path is bubblefree. The presence of the bubble, however, allows a steady state to be achieved sooner and keeps the system cleaner (microbubbles and particulates are continually swept away and do not accumulate in the light path).

\section{INSTRUMENTATION}

The central module is a diode array spectrophotometer adapted for use with fiber optics. It is controlled by a computer that also provides timing for an autosampler and a chromatography-type valve. Liquids are moved by a multichannel pump located outside the glovebox. All of these commercially available modules, some of which have been modified for optimized performance, are described below.

\section{A. Spectrophotometer}

This module is a Hewlett Packard diode array spectrophotometer (model 8452A) that covers the spectral range of $190-820 \mathrm{~nm}$ in 2-nm increments. Since the portion of the spectrum we required is $400-700 \mathrm{~nm}$, the UV-producing deuterium lamp was not needed. Indeed, its output of visible light was deemed marginal. Accordingly, it was replaced with a high intensity tungsten halogen lamp (Gilway, model L104). A special lamp mounting was constructed to position the bulb precisely where the deuterium lamp's arc would have been, and to provide ventilation to conduct its considerable heat away. A source of the required constant voltage was found within this module's original power supply.

\section{B. Pump}

The multichannel peristaltic pump (Alpkem, model 302) was not modified. However, an external $12 \mathrm{~V}_{\mathrm{dc}}$ power supply was obtained to operate the air valves and their circuitry to introduce bubbles into the carrier stream with correct phasing. 


\section{Autosampler}

This unit (ISCO, model "Isis") did not require any modification except the installation of a Kel-F sampling probe (from Alpkem) in place of the supplied stainless steel one that would deteriorate in the $2 \mathrm{~N}$ acid in which standards and samples are prepared. A straightforward electrical connection to a receptacle on the spectrophotometer permits the software to control its various actions: probe up or down, probe to sample or wash station, and tray advance.

Many other commercial samplers are provided with covers to limit evaporation from sample containers and otherwise protect these vessels from becoming contaminated. Therefore, a cover was designed for the ISCO unit, but it was never actually used. Evaporation would likely have been slowed considerably, but there are alternatives such as a software correction based upon the insertion of quality control $(\mathrm{QC})$ samples whose changing observed concentration can be compared with a known initial value.

\section{Flowcell and its Fiber Optics}

Flowcells from Alpkem were available in 3-, 10-, 15-, and 30-mm pathlengths. Early studies indicated a need for a 5-mm pathlength to keep the expected plutonium concentrations from producing absorbances $>1.0$ absorbance units. Accordingly, Vitro Technology fabricated $5-\mathrm{mm}$ flowcells for us. And Alpkem's 30-mm flowcell works well when lower concentrations of plutonium are encountered. Both sizes have a $1.0-\mathrm{mm}$ inside diameter, and light is directed in or out by means of sapphire rods with optically flat surfaces.

Fiber optic cables are readily available, and we selected a pair with a 600-micron core and the standard SMA end fittings (Ensign-Bickford). At the flowcell end, plastic tubing was used initially as a bushing to make the fiber optic endpiece have the same outside diameter as the flowcell, then the two units were connected with plastic sleeving. Later, an adaptor was machined which accepted both the flowcell (held in place with a set screw) and the fiber optics threaded SMA endpiece.

The spectrophotometer's normal configuration includes a platform and spring-loaded holder for a standard $1 \times 1 \mathrm{~cm}$ optical cell. The holder was replaced with a combination of front-surface mirrors and $x-y$ stages (Edmund Scientific) and tube-mounted lenses (Atlantic Industrial Optics). The light beam (normally collimated) is intercepted by the mirror, redirected $90^{\circ}$ to the light path to the lens, and focused on the end of the fiber optic cable distal to the flowcell. The reverse of this optical arrangement returns the light from the 
flowcell to the spectrophotometer's detector compartment. All this is depicted in Figure 3. With proper alignment, the detector doesn't "know" that the

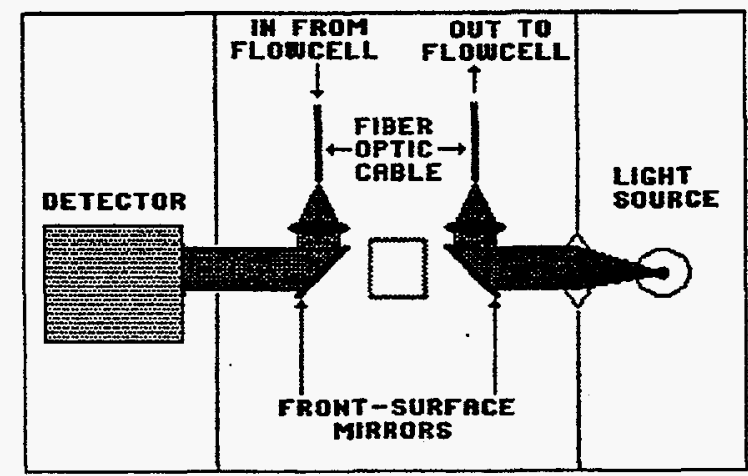

Figure 3. Modification of HP8452.

light beam has been diverted through a remotely located flowcell. Maximum light energy is obtained by using Hewlett Packard's standard package in "kinetic" mode, making sure the flowcell is filled with water and is bubble-free, and tweaking the mirror positioners until minimum absorbance (maximum intensity) is observed. The efficiency of this arrangement is such that we sometimes got the enviable message "too much light" and actually had to defocus a bit.

\section{E. Computer}

Any IBM PC (or compatible clone) can be used if it's fast enough to handle data at the spectrophotometer's maximum rate: 10 data points per second. This means a model 386 or higher.

Our software has been written in QuickBASIC (version 4.5), which is compatible with Hewlett Packard's software library, so that existing routines can often be used without modification. The interface board (HPIB) within the computer accommodates the information exchange with the spectrophotometer and the two external devices plugged into it (ISCO autosampler and Valco valve). A portion of the software program is included in Appendix A to this report.

\section{HYDRAULICS}

In SFA it is customary to plumb the various modules such that fluid flow is right-to-left. Since the principles of SFA as applied to plutonium analysis have been described elsewhere, ${ }^{6}$ only the final version of the hydraulics is presented here. Reference to Figure 4 will make the following description clear. 


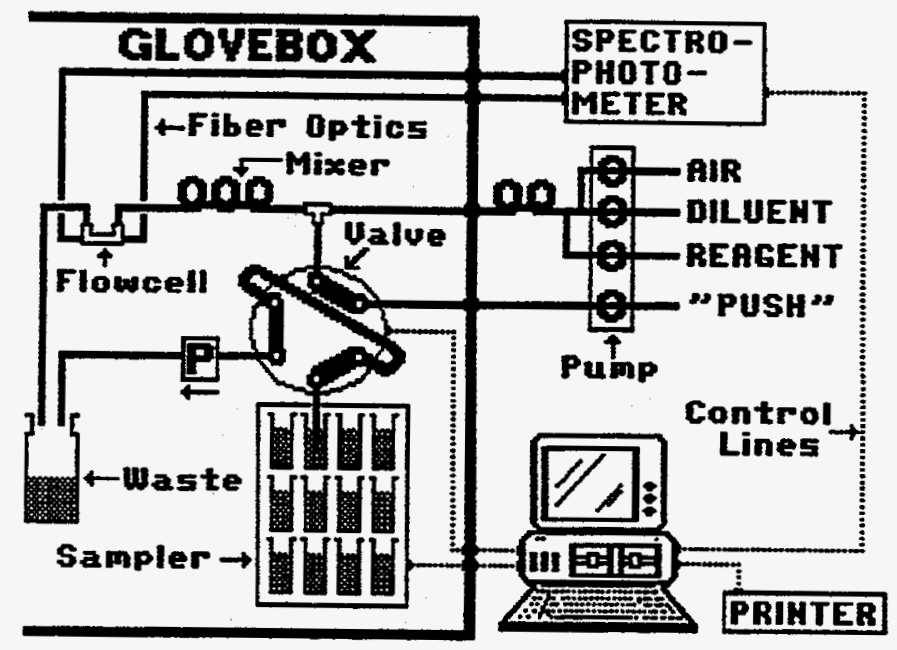

Figure 4. Hydraulics of the Pu analyzer.

The hardware is separated into two groups: those modules that will come into contact with radioactive solutions and will become contaminated and those that can remain outside the glovebox where they will be far easier to modify, adjust, and receive periodic preventive maintenance.

\section{A. Inside the Glovebox}

1. Whenever the $\mathrm{Pu}$ analyzer is in use, its autosampler contains open vials of standards and samples; contamination is almost a certainty, and therefore the autosampler must be inside the glovebox.

2. Since standards or samples must be pumped into the air-segmented stream of reagent at a precise rate despite their not passing through the multichannel pump, they are "stored" temporarily in the external loop of a 6-port, 2-position electrically driven valve (Valco, model C22). A tube (labeled PUSH) from the external pump pushes the contents of the loop into the reagent stream for mixing and passage to the flowcell. This valve, then, contains radioactive solutions and must be inside the glovebox.

3. A small, inexpensive single-channel peristaltic pump (labeled " $P$ " in Figure 4) is used to aspirate samples, standards, or wash solution from the autosampler through the loop and into a waste vessel. Whatever isn't captured inside the loop passes through this pump, and has the potential of contaminating it. 
4. When the flowcell is first used, the inside surfaces clearly become contaminated. Therefore, the flowcell and the attached fiber optics necessarily remain inside the glovebox, also.

\section{B. Outside the Glovebox}

1. The computer and its associated modules (monitor, keyboard, and printer) stand no chance of becoming contaminated, and therefore reside outside.

2. The spectrophotometer can remain outside since it receives absorbance information via fiber optic cables that are sealed into the side of the glovebox.

3. Initially, to keep hydraulic conduits as short as possible, the multichannel pump was placed inside the glovebox. Later, the pump was moved outside the glovebox for two reasons: the pump was a relatively expensive unit and would probably become contaminated, and because maintenance, including pump tube replacement, proved difficult for operators wearing required two pairs of gloves. An equivalent hydraulic circuit was created inside using a chromatographytype valve.

\section{PRE-OPERATION}

To obtain high precision from the $\mathrm{Pu}$ analyzer, attention must be paid to a number of details: number, quality, and concentrations of standards; reagent preparation; instrument warm-up time; bubble pattern of segmented stream; and evaporation control or correction. These are addressed separately below.

\section{A. Standards}

In order for the built-in statistics to produce the best standard curve possible, five levels of standards are recommended. If a single standard is used, the system will generate a straight line between it and an assumed value of zero for the baseline. If two standards are used, zero will not be assumed and the calibration line will be between the two values defined. For three or more levels, a least squares linear fit will be done through all the points (including a zero level if it is identified). While the original design used five levels covering the concentration range of $40-240 \mathrm{~g} / \mathrm{L}$, plus a zero level, the operator should choose standards based on the expected concentrations of the samples to be analyzed. A typical standard curve in our laboratory included these levels: $0,25,75,125,200$, and $250 \mathrm{~g} / \mathrm{L}$. Standards are prepared in $2 \mathrm{~N} \mathrm{HCl}$, and should 
not be more than three months old. If particles are visible, standards should be discarded. Once prepared, standards should be kept in tightly capped vials, else evaporation will cause concentrations to increase with time.

\section{B. Reagents}

Only two solutions must be prepared: a wetting agent and ascorbic acid. The wetting agent (one is nearly always used in SFA) is the surfactant Brij-35. Eight drops of a $35 \%$ solution in a liter of deionized water is labeled "Brij water" and is used in the preparation of everything else.

The concentration of ascorbic acid takes into account the highest expected concentration of the samples and the on-line dilution of both samples and reagent. Twice the stoichiometric amount is used, and for $\mathrm{Pu}$ (IV) diluted 21 -fold, this computes to $8.8 \mathrm{~g}$ of the anhydrous powder in $500 \mathrm{~mL}$ of $2 \mathrm{~N} \mathrm{HCl}$ (diluted with Brij water). Ascorbic acid may be kept for about one week, after which it darkens and raises the instrument's baseline.

\section{Instrument Warmup}

Hewlett Packard's recommendation that the HP8452 diode array spectrophotometer be allowed to warm up for one hour before use was confirmed as being necessary and sufficient. While it is warming up, other operations can be performed, e.g., reagent preparation, establishing hydraulic bubble pattern, and the bookkeeping associated with the samples to be analyzed.

\section{Bubble Pattern}

SFA works well only when the bubble pattern is regular. It is imperative that the pattern be visibly flawless from its point of generation (where air in introduced into the first liquid stream), through the mixing coils, the 6-port valve, and the flowcell. Some factors which affect the flow of a segmented stream include dirt in the system, incorrect connections (annular spaces) between conduits or fittings, and incorrect level of wetting agent. New peristaltic pump tubes will change somewhat during their first hour of operation, but will smooth out after that and remain fairly constant for several hundred hours of operation. This assumes, of course, that the pump's platen is released whenever the pump is off, otherwise flat spots will form on the pump tubes and pumping rates will change. 


\section{E. Evaporation of Standards and Samples}

Inside the glovebox the air is moving rapidly, and this promotes evaporation of all liquids with any surface area exposed to the moving air. Consequently, all standards and sample vessels should remain tightly capped during storage and while on the autosampler. The exception, of course, is during a run when caps must be off. However, to minimize evaporation (and consequent concentration increase), caps can be removed just before the run is initiated and put back on as soon as the last vessel has been sampled.

The algorithm accommodates QC samples to provide a correction for unavoidable evaporation during the run. QC samples (typically equal in concentration to the highest standard, although any standard can be used) can be positioned anywhere on the sample tray, and used as frequently as desired, as long as they are properly identified during the instrument-operator dialog at startup time.

\section{F. Loading the Autosampler Tray}

The instrument is self-calibrating, and as such requires a set of standards preceding the samples to be analyzed. The standards are generally placed on the tray in ascending order (although any known order is OK), followed immediately by the samples in the order that will be presented to the instrument during the startup dialog (see below). The range of standards should always equal or exceed the range of samples, since answers produced by extrapolation are less accurate than those calculated by interpolation. However, the system has been shown to be linear up to $250 \mathrm{~g} / \mathrm{L}$ in the $5-\mathrm{mm}$ flowcell when the dilution factor is 20 .

\section{OPERATION OF THE ANALYZER}

A computer program "PU3" is run either as the stand-alone program "PU3.EXE" or within QuickBASIC as "PU3.BAS". In either case, it begins with a brief dialog in which parameters and questions are displayed on the computer's screen and answers are provided by the operator via keyboard entries. The initial display will tell the operator something about system operation and give the operator an opportunity to abort the run if certain parameters seem unacceptable. For example, the INTENSITY of the source and the DARK CURRENT from the previous run will be displayed and can be compared with the same parameters for the current run. If the INTENSITY of the light beam (making a round trip from the HP8452 light source, through the fiber optic cables and flowcell, back to the instrument's detector) is too low, this may indicate a dirty flowcell, failing light source, or misalignment of one or more parts of the optical system. 
If the INTENSITY is higher or lower than that of the previous run, the GAIN is adjusted automatically by the program. The GAIN can have values in the range of 0 to 15 , but is usually close to one. While operation at higher values is acceptable, rising values indicate a pending problem and should be addressed before another run is made.

Next comes the dialog. The system asks "How many standards?" and expects a response on the keyboard. For each standard, it then requests a concentration value; this is usually given in $\mathrm{g} / \mathrm{L}$. When these numbers have been entered, the next question is "How many samples?" For each sample, the operator has the option of entering some alphanumeric identification, ignoring it by pressing the <ENTER> key, or entering the letters " $Q C$ " if a $Q C$ sample is to be included with the samples on the sampler's tray.

Upon completion of the dialog, the computer screen darkens and then produces a 3-section display that remains for the duration of the analysis. The upper left quadrant confirms some of the operator's entries, including name, number of standards, number of samples, and the name of the file where data are to be stored during the run. The upper right quadrant is reserved for the full scan (absorbance vs wavelength) of the spectrum of each standard and sample. The first tracing occurs when the first standard has passed through the flowcell, and each tracing remains for a full cycle (typically 90 seconds) until replaced by the following scan. The bottom half of the screen shows (in realtime) the absorbance of whatever is in the flowcell at the time. This is an absorbancevs-time plot of the difference between the absorbances at 600 and $640 \mathrm{~nm}-$ essentially, a monitoring of the progress of the analysis. The beginning screen is shown in Figure 5.

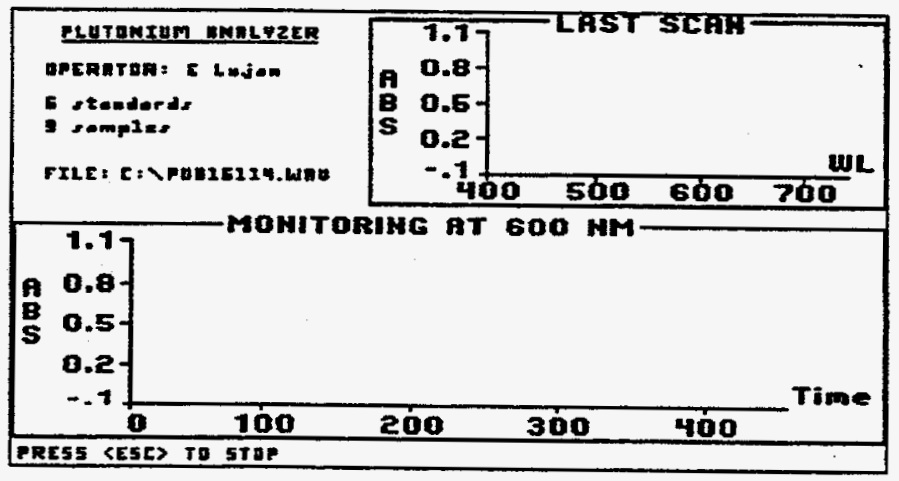

Figure 5. Initial display on the screen.

The tracing begins at time $=0$ and absorbance $=0$. A data point is displayed every two seconds, and points are connected to create a line showing the progress and history of flowcell activity. Beginning immediately, the baseline is checked for linearity. If it is outside preset limits (about $0.1 \%$ RSD), an error message will be displayed and the linearity check will be restarted. This is most 
likely to occur if or when the system is restarted too soon after some change is made (e.g., renewing the ascorbic acid reagent, or replacing a worn-out pump tube). Until the baseline is acceptable to the system, the sampler's probe will not move out of the wash vessel. The events that follow distinguish the two modes of operation: CALIBRATION and ANALYSIS.

\section{A. Calibration}

After all conditions have been satisfied, the sample probe moves out of the wash vessel and into the vial at the sampling position on the autosampler. Liquid, preceded by the air bubble that results when the probe is out of a liquid (i.e., in the air between wash and sample), is aspirated by the small singlechannel peristaltic pump towards the 6-port valve. Just before the leading air bubble reaches the valve, a timing signal switches the valve from INJECT to LOAD, thus directing the sample plug (with its flanking air bubbles) into the external loop of the valve. When the sample is totally within the loop, the valve is switched from LOAD to INJECT, and the sample plug is pushed out of the loop (by the pump tube labeled PUSH), through the T-fitting, and into the air-segmented stream of reagent. The sample then flows through the mixing coil(s), through the flowcell, and to waste.

When the leading edge of the reaction mixture (which now contains some sample) reaches the flowcell, the absorbance will increase in proportion to the sample or standard's concentration, and this will appear as a rising tracing on the lower half of the screen. If the first standard is a zero level, there will be no apparent change in the tracing. When steady state is reached, the tracing will become relatively flat for about 40 seconds; then it will return to the baseline as the sample is washed out of the flowcell. In the middle of this steady state condition, quantitation will be invoked for precisely 24 seconds ( 36 segments). At the end of this data gathering period, the tracing will display (in a contrasting color) the absorbance of the sample (at the monitoring wavelength) and its position in time. The upper right quadrant of the screen will also display the entire spectrum over the range of $400-700 \mathrm{~nm}$. All this is shown in Figure 6 .

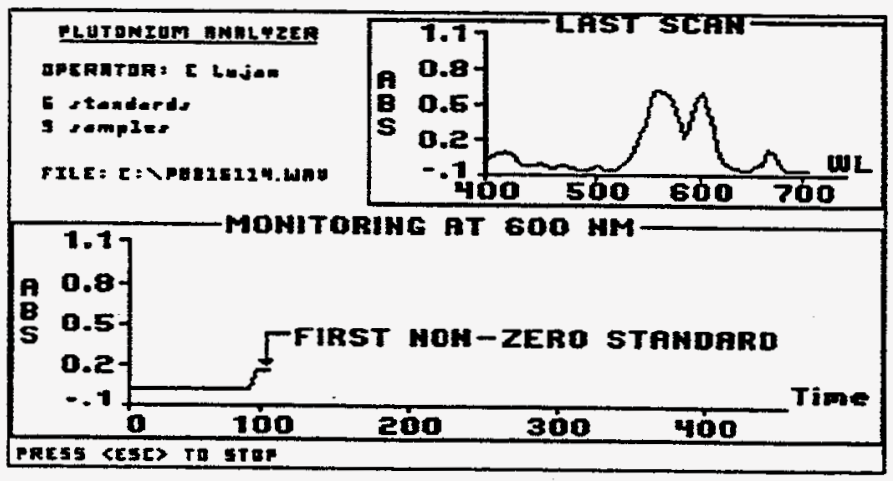

Figure 6. Screen early in the run. 
The analysis proceeds, with the realtime display showing each peak and its full spectrum. During the calibration, while standards are being analyzed, the realtime tracing is colored red and the quantitation line is displayed in yellow. The quadrant labeled LAST SCAN isn't erased, and each succeeding scan overlays the previous ones so that a family of tracings of standards is visible. This gives the operator assurance that all is well and the system is performing as desired.

When the last standard has been quantitated, the actual calibration procedure is invoked. The four absorbance differences produce four separate regression lines, and these are displayed in the upper left quadrant, replacing what was there previously. These lines are color-coded to identify the absorbance differences from which they were calculated. Also, the correlation coefficient (labeled CC) is given for each regression, and these are typically between 0.98 and 1.00 (the latter being a consequence of rounding of numbers $>0.995$ ). If any $\mathrm{CC}<0.98$, the analysis should be restarted because linearity is unacceptable. Figure 7 shows the screen as it appears following the last standard.

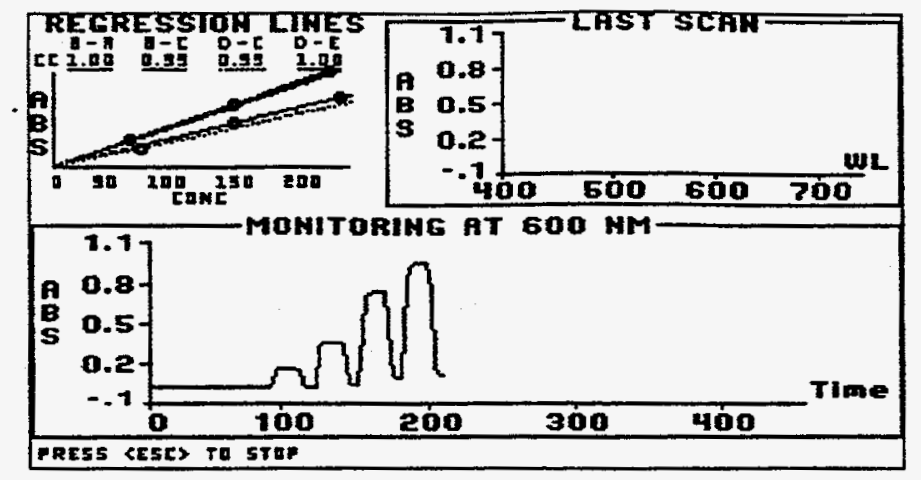

Figure 7. Screen after the last standard.

\section{B. Analysis}

When the tracing of the realtime monitoring returns to baseline following the last standard, it changes from red to green, thus confirming for the operator that the system is now in its ANALYSIS mode. When the first sample has been quantitated and its spectrum displayed, the upper left quadrant changes again into a real-time display of answers. As shown in Figure 8, each sample is listed with its alphanumeric ID (if one was provided during the initial dialog), its concentration, and the standard deviation (SD) of the four intermediate concentrations derived from the four separate regression lines. After eight samples have been displayed, the listing scrolls upward such that the last eight are always in view. 


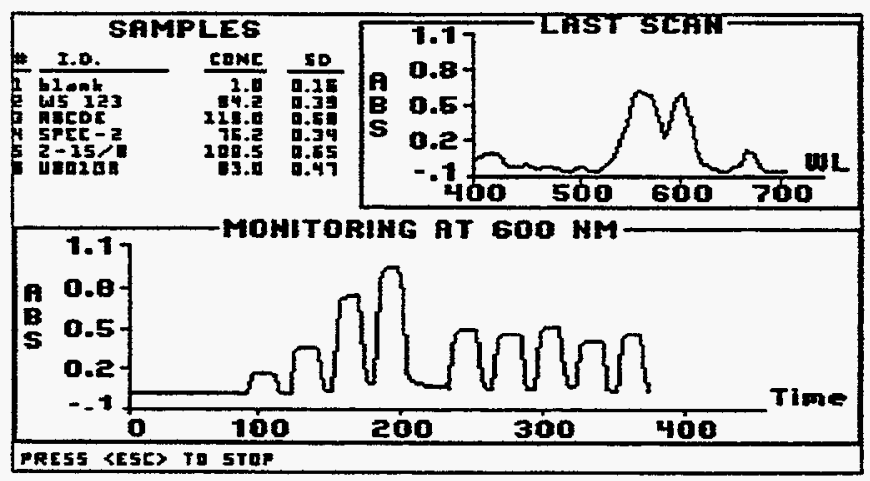

Figure 8. Screen during analysis.

\section{C. $\underline{\text { Printout }}$}

After the final sample has been analyzed, hard copy is produced on the attached printer. A sample printout is reproduced in Appendix B. Its heading gives the date, time, operator, and the name of the file where the data were stored.

For each standard used, the printout presents (1) a comparison of the given value with what was calculated from the regression lines, and (2) the calculated standard error of estimate (SEE) for the calibration. Then, for each of the absorbance differences, the printout shows the intercept, slope, and CC associated with the regression line. Finally, the printout indicates the weighting of each regression based upon the residuals of the data points used in calculation. These weights are used in the alternate calculation of averages for each sample concentration.

For each sample, the ID is given along with the individual. concentrations from each regression, the average and $\mathrm{SD}$, the weighted average, and the standard error of performance (or standard error of prediction) (SEP).

Finally, if replicates are present, additional statistics are printed: the number $(N)$ in each group of replicates, average and SD, and, if N>2, precision (as \% RSD).

\section{RESULTS}

An early comparison of the automated instrument with radiochemistry is shown in Figure 9. The correlation $\left(R^{2}=0.997\right)$ between the two methods prompted us to continue efforts to establish where errors were occurring and how they could be minimized. 


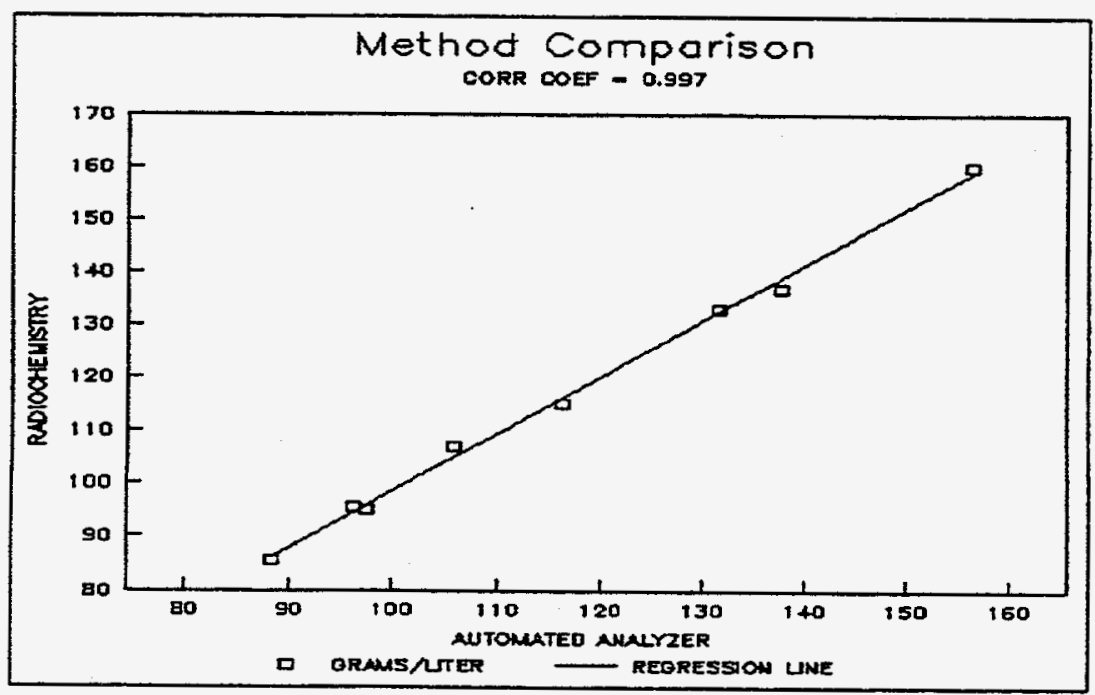

Figure 9. Automated analyzer vs radiochemistry.

An exhaustive study of all controllable factors revealed two major sources of irreproducibility: system drift and peristaltic pump noise. These are addressed separately below.

\section{A. System Drift}

When the same sample is analyzed repeatedly, the reported concentration rises steadily. This can be traced to evaporation promoted primarily by the rapidly circulating air inside the glovebox. Under these windy conditions, evaporation has been shown to account for liquid loss of about $3 \%$ per hour-more from sample vessels with larger diameters and/or those that are filled closer to the top. A correction is possible if QC samples are inserted at regular intervals (e.g., every sixth position). Figure 10 shows the effect of such a correction using neodymium $(\mathrm{Nd})$ as a stand-in for plutonium.

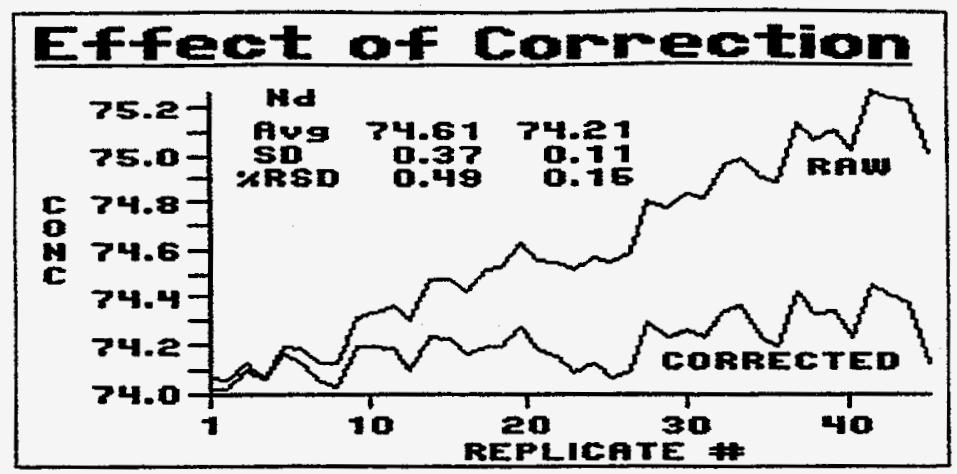

Figure 10. Effect of correction using interspersed QC samples. 


\section{B. Roller Noise}

The nemesis of all peristaltic pumps is the "unavoidable" hydraulic noise introduced by each roller as it lifts off the compressed pump tube and superimposes a slight reverse flow upon the larger forward flow. On multichannel peristaltic pumps, this effect is often said to be "identical" for each pump tube, so the overall proportioning is nearly constant. Accordingly, in SFA the composition of each segment in a stream resulting from the combination of two or more pump tubes is presumably the same.

When hydraulic debubbling is used, the effect is further damped by mixing inside the debubbler; the segmented stream emerges from the debubbler as an unsegmented stream. But the advantages of bubble through the flowcell outweigh this smoothing effect as was stated earlier (e.g., faster attainment of steady state, a cleaner system, smaller sample size, and faster system operation).

Given that the software must somehow ignore the bubbles as they pass through the flowcell, it might appear at first glance that the data obtained are essentially the same. Figure 11 compares the observed intensity of energy passing through

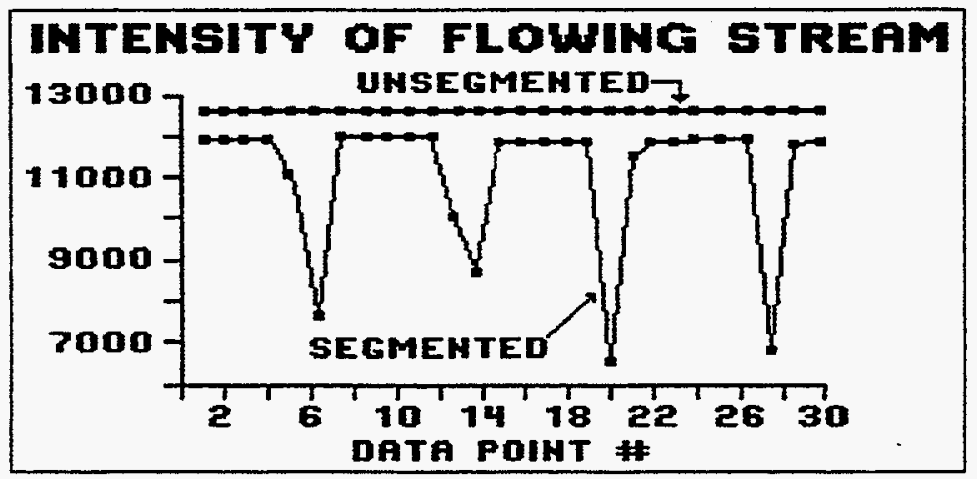

Figure 11. Segmented vs unsegmented flow.

the flowcell for both segmented and unsegmented streams. Needless to say, when the bubble interrupts the light beam, there is a drastic decrease in transmitted light, and a downward dip occurs in the tracing. When the plateaus at the top of the segmented stream are scrutinized, values for each segment aren't as invariant as they first appear. These individual data points were collected at $100 \mathrm{msec}$ intervals (ten per second), and at a rate of 90 bubbles per minute ( 90 segments per minute), one sees three segments every two seconds.

The algorithm we use begins by identifying the first "minimum" it can find-a value which is lower than the two values on each side of it. With this as a starting point, it then finds the fourth minimum, thus isolating three segments as shown in Figure 12. While these plateaus appear to have identical 
segments as shown in Figure 12. While these plateaus appear to have identical intensities, an expansion of the scale (see Figure 13) reveals that each segment has a slightly different maximum. These three maxima are retained for eventual calculations, but are averaged to produce a single point for monitoring.

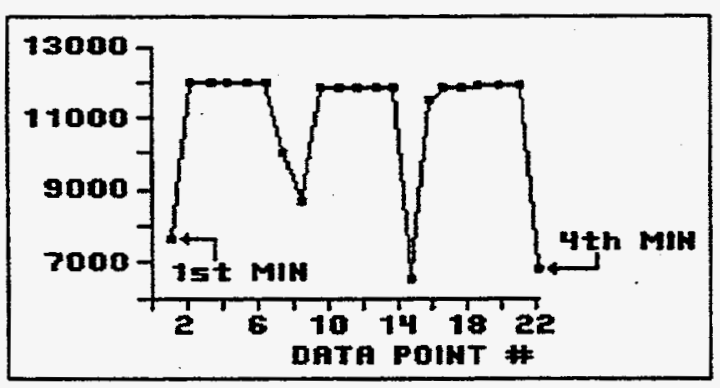

Figure 12. The first 3 segments on a normal scale.

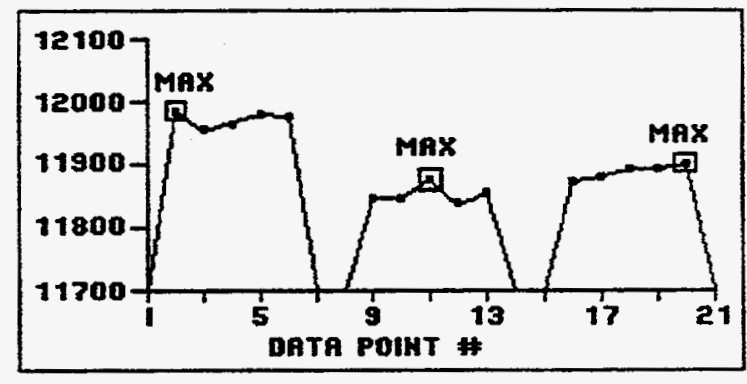

Figure 13. Maxima on an expanded scale.

If one now plots 36 of these maxima (representing 36 segments in 24 seconds), the line isn't as straight as one would like (see Figure 14). Since the peristaltic pump has 12 rollers, 36 segments (one per roller)

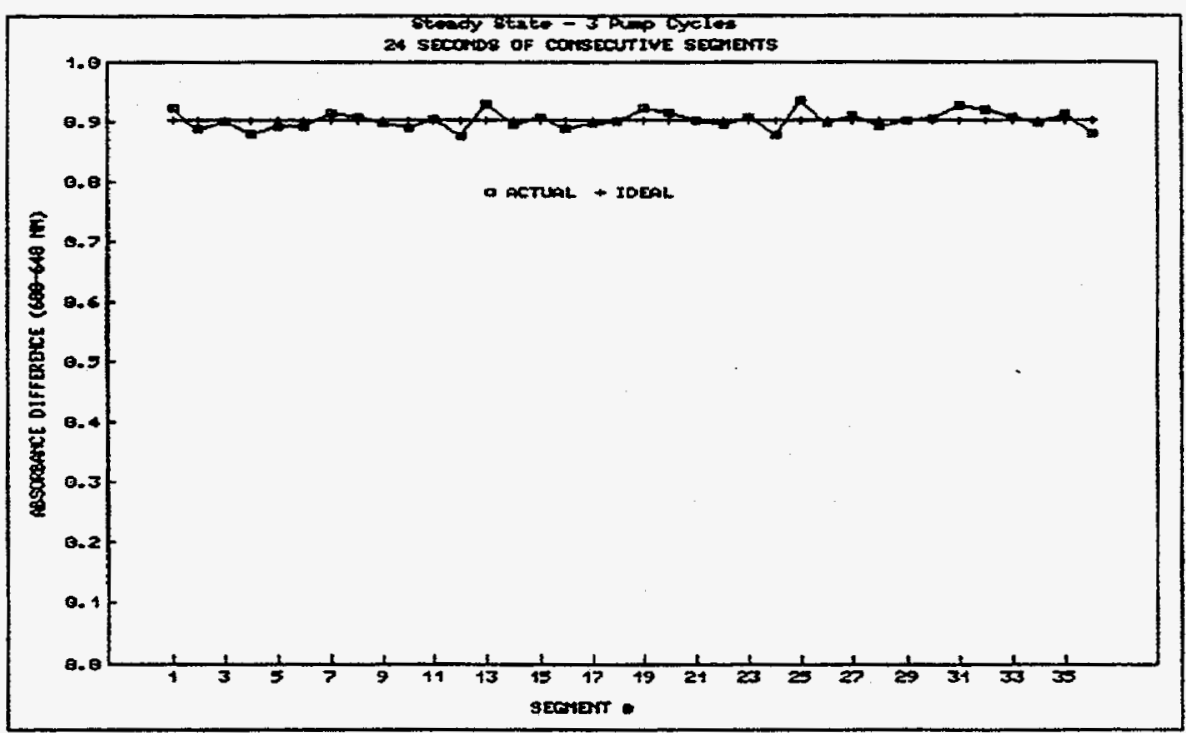

Figure 14. Thirty-six maxima on a normal scale

represent exactly three rotations of the pump. When the scale is expanded, as in Figure 15, the repeating pattern of the three pump cycles emerges, confirming that the 12 segments within a single pump cycle are not identical. This knowledge is used later to remove occasional (rare) "bad" segments such as might be caused by particulates or microbubbles. For example, 


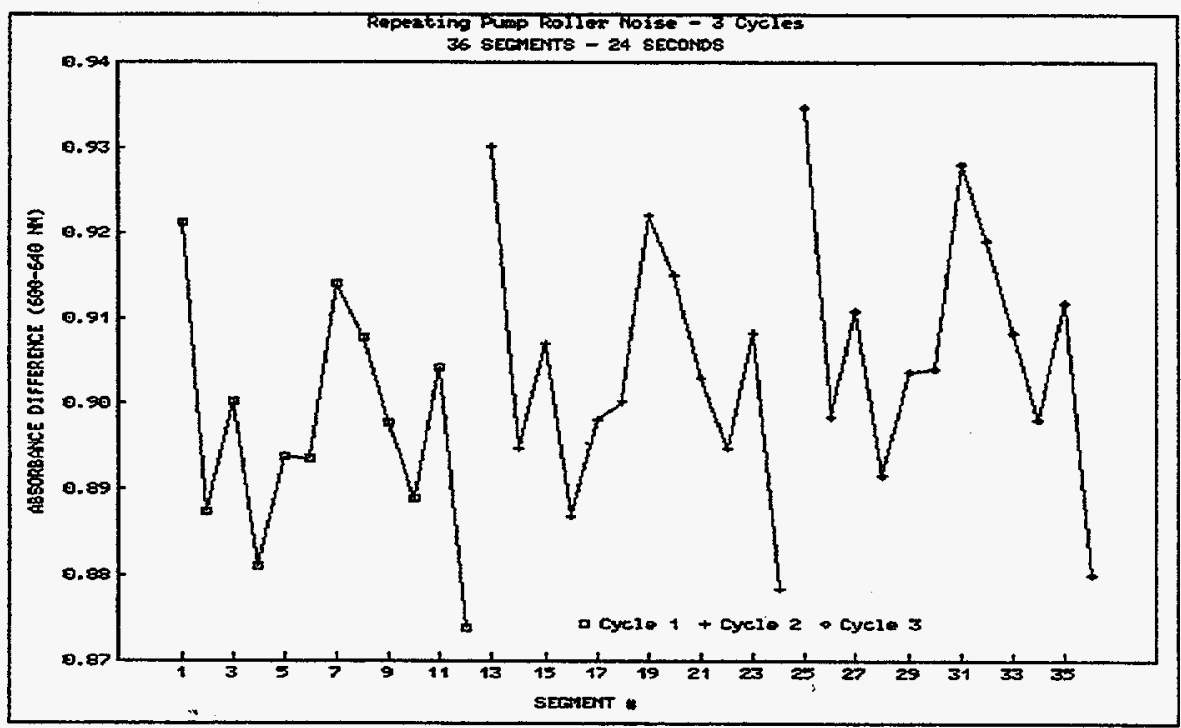

Figure 15. Thirty-six maxima on an expanded scale.

Figure 16 depicts an abnormally high absorbance resulting from an unwanted particle in a particular segment. The algorithm determines which segment is "bad," then identifies the equivalent segments in the other two pump cycles. These are averaged to provide a replacement value for the bad segment, thus providing an overall better group of 36 than would otherwise prevail (see Figure 17). Clearly, an uncorrected value for a bad segment would bias a least squares linear fit through the group of 36 values.

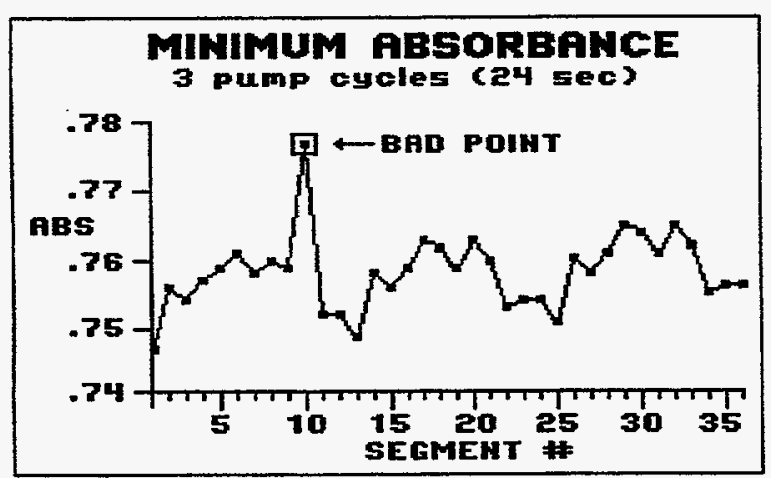

Figure 16. Single bad segment.

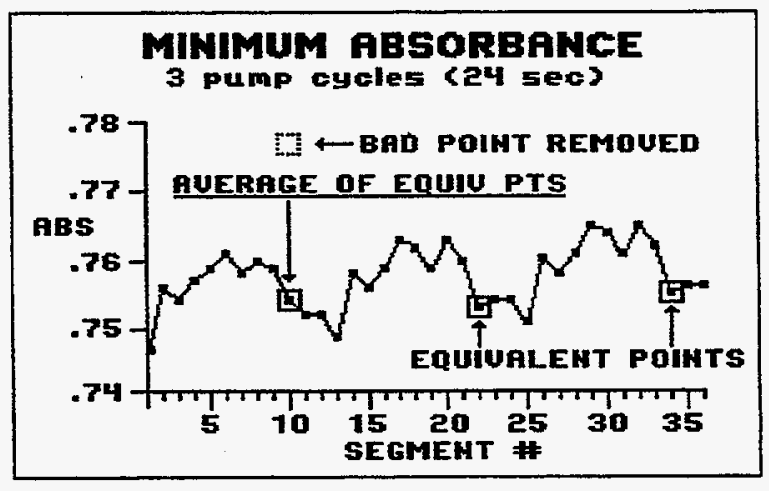

Figure 17. Corrected segment.

\section{Final Version}

The final study involved operating the instrument on three nonconsecutive days using as "truth" meticulously prepared standards at three different levels: 50,75 , and $100 \mathrm{~g} / \mathrm{L}$. These given values were compared with observed values, 
and the graph is reproduced as Figure 18 below. The associated statistics are as follows:

$$
\begin{gathered}
\mathrm{R}^{2}=0.9994, \\
\text { slope }=1.019, \text { and } \\
\text { intercept }=-1.309 .
\end{gathered}
$$

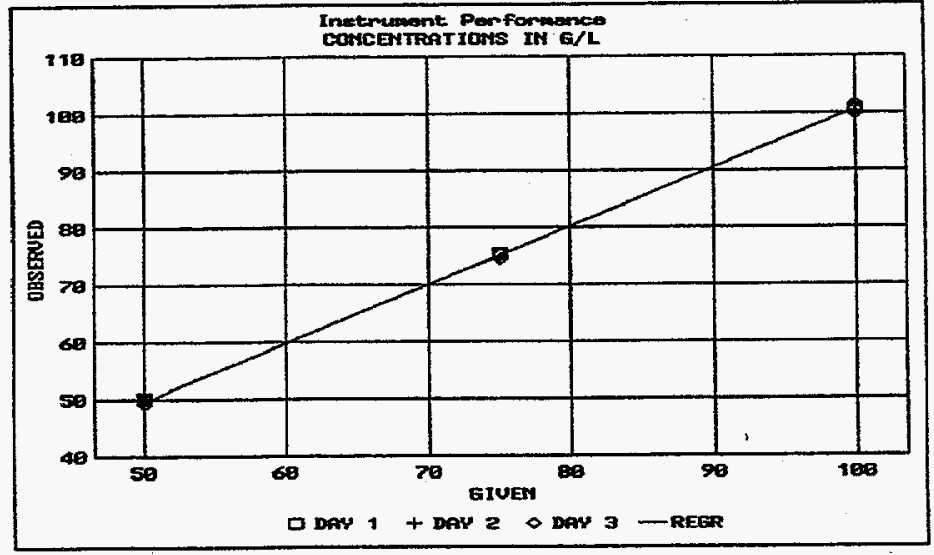

Figure 18. Instrument performance.

The last activity was to compare the automated plutonium analyzer with the manual method that it would likely replace. For this comparison, 36 standards were prepared and half used on each instrument. Each group of 18 was divided into 9 to establish the standard curves and 9 "unknowns" for the direct comparison. Figure 19 is a graph of the results.

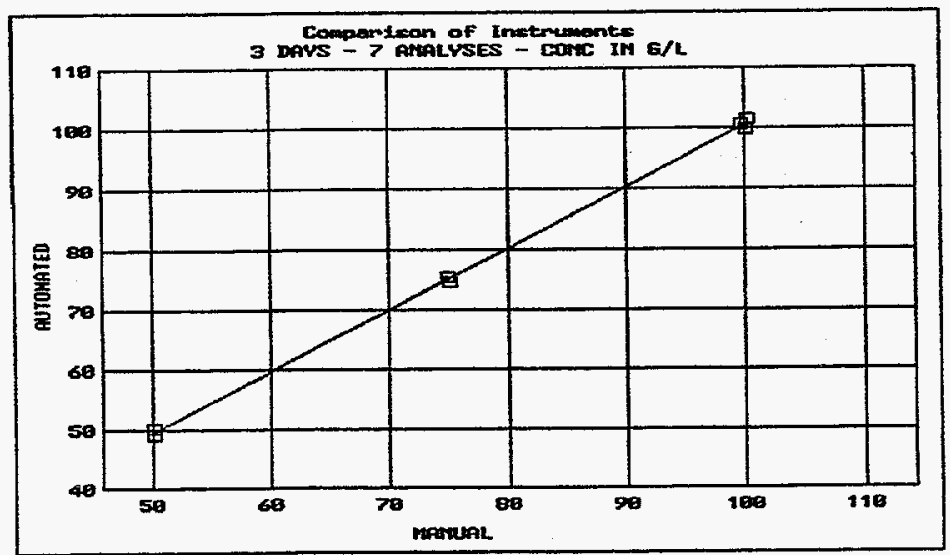

Figure 19. Instrument comparison.

\section{Optional High-Sensitivity Version}

By making certain changes in the hydraulics, we have demonstrated a sensitivity improvement of nearly two orders of magnitude. The two 
figures below depict the hydraulic or optical changes: larger sample loop, longer flowcell, and pump tube replacements to change the dilution factor from 20 to 1.7 .

Figure 20 (similar to Figure 4) shows the manifold used in all work previously described, while Figure 21 shows the modified

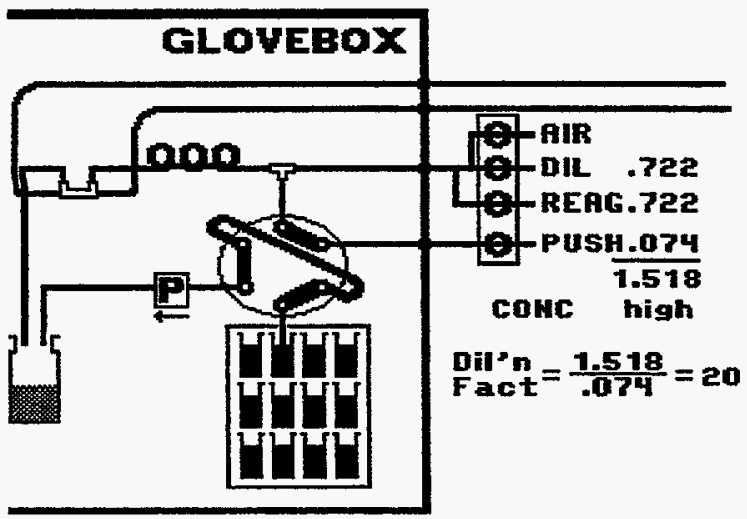

Figure 20. 20x dilution.

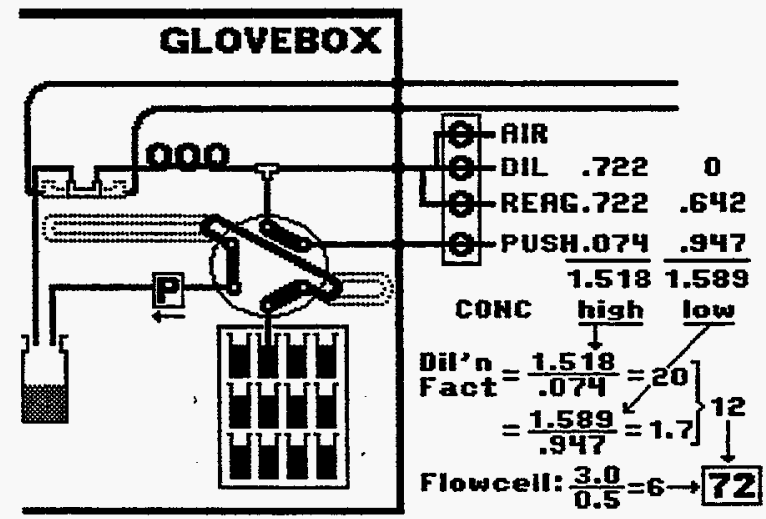

Figure 21. 1.7x dilution.

manifold that improves sensitivity by a factor of 72 . The numbers opposite the pump tubes represent flow rates in $\mathrm{mL} / \mathrm{min}$ for the high and low levels of analyte. The revised manifold was tested with plutonium levels in the range 0.6 to $4.4 \mathrm{~g} / \mathrm{L}$, and a typical family of tracings is shown in Figure 22. The corresponding regression lines for three of the four absorbance differences are shown in Figure 23.

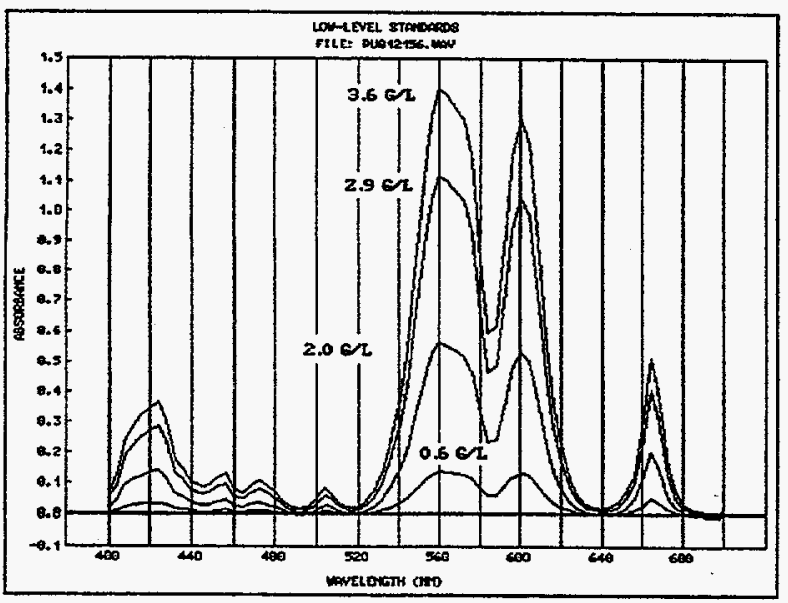

Figure 22. Low-level standards.

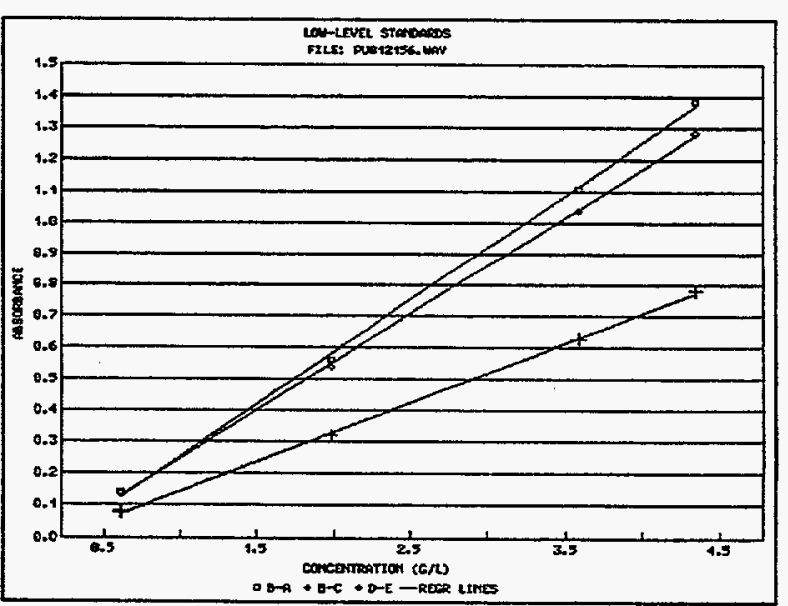

Figure 23. Regression lines. 
Correlation coefficients all exceeded 0.999 , and replicate determinations produced the following statistics:

$\begin{array}{lllll}\text { ID } & \underline{\mathrm{N}} & \underline{\text { AVERAGE }} & \underline{\text { SD }} & \underline{\text { RSD }} \\ \text { A } & 4 & 0.642 & 0.001 & 0.12 \% \\ \text { B } & 5 & 1.943 & 0.002 & 0.12 \\ \text { C } & 5 & 3.562 & 0.006 & 0.18 \\ \text { D } & 5 & 4.379 & 0.006 & 0.14\end{array}$

\section{CONCLUSIONS}

The spectrophotometric analysis of plutonium solutions has been totally automated using segmented flow analysis and a design that provides nearly a 10 -fold improvement in precision over the usual wet chemical analysis. Using an on-line reduction of all $\mathrm{Pu}$ to $\mathrm{Pu}(\mathrm{III})$, quantitation is based on absorbance differences from data gathered at steady-state via fiber optics at five discrete wavelengths. Each analysis requires only $0.074 \mathrm{~mL}$ of sample or standard and generates about $1.5 \mathrm{~mL}$ of waste. The system operates unattended at 40 samples per hour and is self-calibrating. Its precision of $0.05 \%-0.20 \%$ RSD is a consequence of an algorithm that addresses pump roller noise and correction for evaporation through periodic insertion of QC samples. Ths system was designed for operation with a glovebox, but only an autosampler, a loop valve, an inexpensive single-channel pump, and a flowcell must be inside the glovebox. Major modules (spectrophotometer, multichannel peristaltic pump, and controlling computer) remain outside the glovebox.

Hydraulics may be configured to handle plutonium concentrations from 0.4 to $240 \mathrm{~g} / \mathrm{L}$. Reports are generated on site, with the analyte in correct concentration units, and accompanied by an abundance of statistics.

\section{ACKNOWLEDGMENTS}

The efforts of the following scientists are acknowledged for their significant contributions to the design, assembly, operation, and testing of the plutonium solution analyzer: Carolyn Huff (UCLA summer student), Dr. Darryl Jackson (Section Leader), Elmer Lujan (technician), Laurie Walker (chemist), and Dr. Lawrence E. Wangen (Task Area Leader and math and statistics consultant). 


\section{REFERENCES}

1. J. M. Cleveland, "The Chemistry of Plutonium," La Grange Park, Illinois: American Nuclear Society, P. 17 (1979).

2. F. W. Tober and E. R. Russell, USAEC Report DP-349 (February 1959).

3. G. A. Burney and F. W. Tober, Ind Eng Chem Process Design Develop, 4,28 (1965).

4. A. M. Harmon, T. R. Hahn, and T. K. Marshall, "Spectrophotometric Determination of Plutonium in 238Pu Oxide," Pittsburgh Conference, Abstract \#1014 (1984).

5. L. E. Wangen, M. V. Phillips, and L. F. Walker, "Use of Multivariate Calibration for Plutonium Quantitation by the Pu(III) Spectrophotometric Method," Los Alamos National Laboratory document LA-11297-MS, May 1988.

6. D. A. Burns, L. E. Wangen, P. Mendoza, M. Randow, E. Lujan, D. J. Temer, and D. Jackson, "Automated Analysis of Plutonium Solutions Over a Wide Range," Trans, 63, Supplement 1, American Nuclear Society (1991). 
Appendix A

Portion of the Software Program

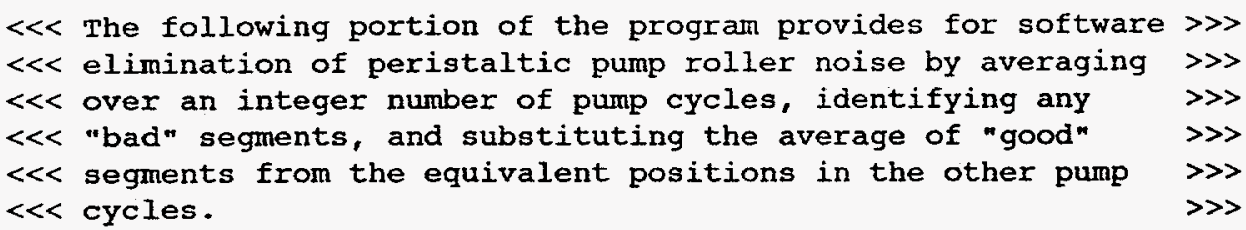

scanit:

IF UVERR THEN STOP

OLDINDEX\% = index?

' Save index8

$s \%=0$

NUMWAVES $=5:$ WAVELIST $(1)=516:$ WAVELIST $(2)=560$

- Reset segment

CLEAR 852

- Reset from 2

CALI UPLOAD

CALL SEND8452("MES")

STDNUMO $=$ STDNUMF +1

- Increment STDNUM8

IF STDNUMO > NUMSTDS THEN SAMPNUM\& $=$ SAMPNUM\& +1

- or SAMPNUM8

FOR $K Z=1$ TO 5

CAII READINFO(DAS)

CONVERTDATA (DAS)

- Read 5 groups

FOR $J=1$ TO 5

- of data points

TABLEMONITOR $(K 8, J)=\operatorname{RAW}(J, 2)$

NEXT $J$

NEXT K8

'Find lst minimum

DO UNTIL TABLEMONITOR(K8 - 3,4) < TABLEMONITOR(K8 - 4, 4) AND TABLEMONITOR(K8 - 3, 4) < TABLEM

- ONITOR(K8 - 5,4) AND TABLEMONITOR(K8 - 3,4) < TABLEMONITOR(K8 - 2, 4) AND TABLEMONITOR(K8

- $-3,4)<$ TABLEMONITOR(K8 - 1,4)

CAILI READINFO (DAS)

CONVERTDATA (DAS)

FOR $J=1$ TO 5

TABLEMONITOR(KZ, J) $=\operatorname{RAW}(J, 2)$

NEXT $J$

$\mathrm{K} \%=\mathrm{K} \%+1$

LCOP

OLDMIN $=\mathrm{K} 8-3$

SUM2 $=0$

FOR GROUP: $=1$ TO 12

TOP $=25$ - OLDMIN $:$ IF GROUP $=1$ THEN TOP $=3$

'Repeat 12 times

FOR R\& $=1$ TO TOP

FOR $J=1$ TO 5

TABLEMONITOR $(K 8, J)=$ TABLEMONITOR $(K 8+$ OLDMIN $-1, J)$

NEXT J

NEXT KO

FOR $K 8=T O P+1$ TO 24

'shift table

CALL READINFO(DA\$)

CONVERTDATA (DAS)

FOR $J=1$ TO 5

TABLEMONITOR(K\&, J) $=\operatorname{RAW}(J, 2)$

NEXT J

NEXT Ko

$\min =1$

SUM $=0$

FOR $N=1$ TO 3

Gosub Findmax

$5 \%=5 \%+1$

FOR $J=1$ TO 5

$K=\operatorname{WAV}(J)$

Complete table

'Find 3 segments

- For each segment

- find max intensity 


\section{Appendix A, continued}

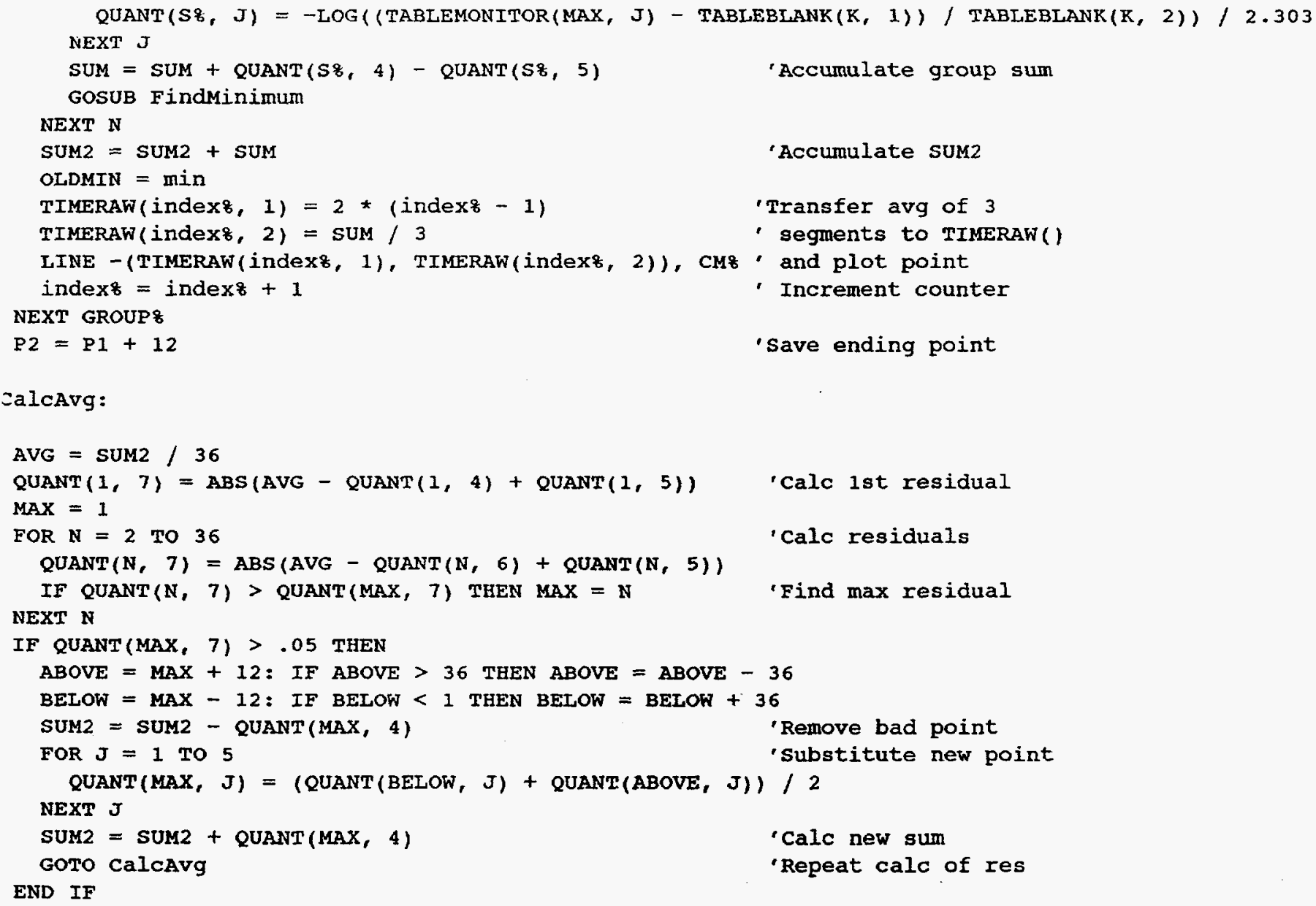




\title{
Appendix B
}

\section{Sample Printout}

\author{
P I U T O N I U M A N A I Y S I S
}

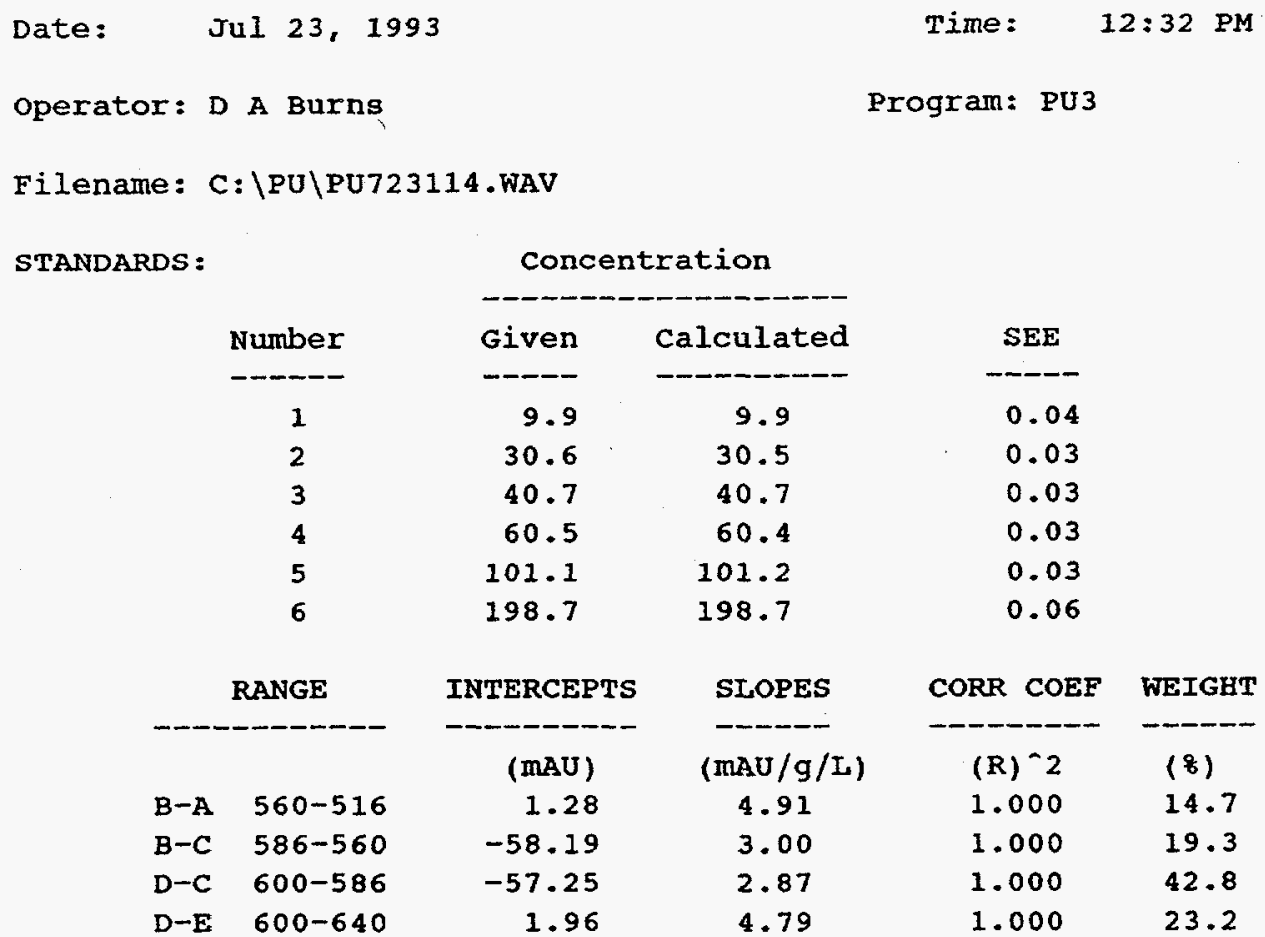

SAMPLES :

\begin{aligned} \# & IDENTIFICATION \\ \hline 1 & A \\ 2 & B \\ 3 & C \\ 4 & D \\ 5 & E \\ 6 & F \\ 7 & A \\ 8 & B \\ 9 & C \\ 10 & D \\ 11 & E \\ 12 & F \\ 13 & A \\ 14 & B \\ 15 & C \\ 16 & D \\ 17 & E \\ 18 & F \\ 19 & A \\ 20 & B \\ 21 & C \\ & \end{aligned}

CONC FROM EACH REGRESSION

\begin{tabular}{|c|c|c|c|}
\hline $\mathbf{B}-\mathbf{A}$ & $B-C$ & $D-C$ & $D-E$ \\
\hline$-\cdots$ & $-\cdots$ & $--\infty$ & \\
\hline 10.80 & 10.87 & 10.61 & 10.65 \\
\hline 30.62 & 30.59 & 30.53 & 30.57 \\
\hline 40.77 & 40.70 & 40.73 & 40.77 \\
\hline 60.57 & 60.46 & 60.63 & 60.67 \\
\hline 101.25 & 101.12 & 101.48 & 101.46 \\
\hline 199.01 & 199.06 & 198.90 & 198.92 \\
\hline 10.75 & 10.85 & 10.58 & 10.62 \\
\hline 30.66 & 30.71 & 30.61 & 30.65 \\
\hline 40.81 & 40.91 & 40.86 & 40.91 \\
\hline 60.71 & 60.70 & 60.82 & 60.84 \\
\hline 101.41 & 101.38 & 101.68 & 101.65 \\
\hline 198.93 & 199.16 & 198.91 & 198.87 \\
\hline 10.82 & 10.87 & 10.63 & 10.68 \\
\hline 30.61 & 30.73 & 30.63 & 30.67 \\
\hline 40.91 & 40.86 & 40.88 & 40.97 \\
\hline 60.77 & 60.74 & 60.89 & 60.92 \\
\hline 101.44 & 101.37 & 101.71 & 101.68 \\
\hline 199.40 & 199.55 & 199.30 & 199.32 \\
\hline 10.88 & 10.96 & 10.70 & 10.74 \\
\hline 30.77 & 30.75 & 30.67 & 30.71 \\
\hline 40.94 & 40.92 & 40.91 & 40.99 \\
\hline
\end{tabular}

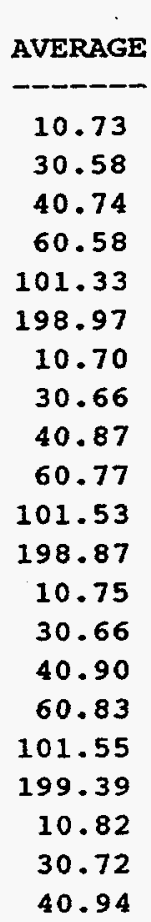

STD DEV

-

0.07

0.02

0.02

0.05

0.10

0.04

0.07

0.02

0.03

0.04

0.09

0.08

0.07

0.03

0.02

0.05

0.10

0.06

0.07

0.03

0.02
WEIGETED AVERAGE SEP

$10.70 \quad 0.11$

$\begin{array}{ll}30.56 & 0.11\end{array}$

$40.74 \quad 0.11$

$60.60 \quad 0.11$

$101.37 \quad 0.11$

198.950 .12

$10.67 \quad 0.11$

$30.65 \quad 0.11$

$40.87 \quad 0.11$

$60.79 \quad 0.11$

$101.58 \quad 0.11$

$198.95 \quad 0.12$

$\begin{array}{lll}10.71 & 0.11\end{array}$

$30.66 \quad 0.11$

$\begin{array}{ll}40.89 & 0.11\end{array}$

$60.85 \quad 0.11$

$101.60 \quad 0.11$

$199.37 \quad 0.12$

10.790 .11

30.710 .11

$40.93 \quad 0.1]$ 
Appendix B, continued

$\begin{array}{ll}22 & \text { D } \\ 23 & \text { E } \\ 24 & \text { F }\end{array}$

$\begin{array}{rrrr}60.75 & 60.71 & 60.84 & 60.89 \\ 101.43 & 101.40 & 101.73 & 101.75 \\ 199.70 & 199.72 & 199.40 & 199.53\end{array}$

60.80

101.58

199.59

0.05

60.81

$0.11 \quad 101.62$

$0.09 \quad 199.54$

0.11

STATISTICS FOR REPLICATES:

IDENTIFICATION
A
B
C
D
E
F

$\begin{array}{cc}\text { N } & \text { AVERAGE } \\ -- & - \\ 4 & 10.8 \\ 4 & 30.7 \\ 4 & 40.9 \\ 4 & 60.7 \\ 4 & 101.5 \\ 4 & 199.2\end{array}$

STD DEV
0.04
0.05
0.07
0.10
0.10
0.27

RSD

0.428

0.178

0.188

0.168

0.108

0.138 